\title{
The Archimedes project: a feasibility study for weighing the vacuum energy
}

\section{E. Calloni ${ }^{*},{ }$ S. Caprara ${ }^{b}$, M. De Laurentis ${ }^{a}$, G. Esposito ${ }^{c}$, M. Grilli ${ }^{b}$, E. Majorana ${ }^{d}$, G. P. Pepe ${ }^{e}$, S. Petrarca ${ }^{b}$, P. Puppo ${ }^{d}$, F. Ricci ${ }^{b}$, L. Rosa ${ }^{a}$, C. Rovelli ${ }^{f}$, P. Ruggi ${ }^{g}$, N. L.} Saini $^{b}$, C. Stornaiolo ${ }^{c}, \mathbf{F}$. Tafuri ${ }^{h}$

a Università di Napoli Federico II, Dipartimento di Fisica and INFN Sezione di Napoli

Complesso Universitario di Monte S. Angelo, Via Cintia Edificio 6, 80126 Napoli, Italy

b Universitá di Roma "La Sapienza" e INFN Sezione di Roma - P.le A. Moro 2, I-00185, Roma, Italy

cINFN Sezione di Napoli Complesso Universitario di Monte S. Angelo, Via Cintia Edificio 6, 80126 Napoli, Italy

d INFN Sezione di Roma - P.le A. Moro 2, I-00185, Roma, Italy

e Università di Napoli Federico II, Dipartimento di Fisica and INFN Sezione di Napoli

Piazzale Tecchio 8080126 Napoli, and Complesso Universitario di Monte S. Angelo, Via Cintia Edificio 6, 80126 Napoli, Italy

f Aix Marseille Université CNRS, CPT, UMR 7332, 13288 Marseille, and Universitè de Toulon, CNRS, CPT, UMR 7332, 83957 La Garde, France

$g$ European Gravitational Observatory (EGO) I-56021 Cascina (Pi), Italy

h Dipartimento Ingegneria dell'Informazione, Seconda Università di Napoli, I-81031 Aversa (CE) and INFN Sezione di Napoli

Complesso Universitario di Monte S. Angelo, Via Cintia Edificio 6, 80126 Napoli, Italy

E-mail: enrico.calloni@na.infn.it, sergio.caprara@romal.infn.it, martina.delaurentis@na.infn.it, giampiero.esposito@na.infn.it, marco.grilli@romal.infn.it, ettore.majorana@romal.infn.it,

gpepe@na.infn.it, silvano.petrarca@romal.infn.it,

paola.puppo@romal.infn.it,fulvio.ricci@romal.infn.it,

luigi.rosa@na.infn.it, rovelli@cpt.univ-mrs.fr,

paolo.ruggi@ego-gw.it, naurang.saini@romal.infn.it,

cosimo.stornaiolo@na.infn.it, francesco.tafuri@na.infn.it

Archimedes is a pathfinder experiment aimed at verifying the feasibility of measuring the interaction of vacuum fluctuations with gravity. The future experiment should measure the force that the earth's gravitational field exerts on a Casimir cavity by using a balance as the small force detector. Archimedes analyzes the important parameters of the future experiment and experimentally explores solutions to the most critical problems.

Frontiers of Fundamental Physics 14 - FFP14,

15-18 July 2014

Aix Marseille University (AMU) Saint-Charles Campus, Marseille

${ }^{*}$ Speaker. 


\section{Scientific motivations}

One of the profound open questions of modern physics is the irreconcilability among the quantum mechanical theory of vacuum and General Relativity. The enormous value of the energy density of vacuum fluctuations as foreseen by quantum mechanics, if inserted naively into the energy-momentum tensor on the right-hand side of the field equations of General Relativity, is not at all compatible with the observed radius of the universe, nor with the acceleration of expansion: a problem known as the cosmological constant problem [1]. At present, in spite of a detailed and important theoretical work [2], there is no general consensus on the theoretical solutions proposed and on the fact that vacuum fluctuations do contribute to gravity [3]. Furthermore, even though the common belief is that this should be the case $[4,5]$, no experiment has been performed to finally verify or discard this assumption. In a recent paper we have shown that considering the present technological achievements on small force detectors, on superconductors and on seismic isolation, it is possible to foresee an experimental path towards such a measurement [6]. The principle of the measurement is the weighing of a Casimir cavity. Indeed, it can be shown that if a Casimir cavity is placed in the earth's gravitational field and the vacuum energy does interact with gravity, it receives a force directed upwards equal to [6]:

$$
\vec{F}=\mathscr{A} \frac{\pi^{2} \hbar}{720 a^{3}} \frac{\mathrm{g}}{c} \hat{z}=\frac{E_{\text {cas }}}{c^{2}} \overrightarrow{\mathrm{g}},
$$

where $\mathscr{A}$ is the Casimir cavity proper area, $a$ is the Cavity proper distance among the plates, $c$ is the speed of light, $\vec{g}$ is the earth's gravitational acceleration ( $\mathrm{g}$ its modulus), the unit vector $\hat{z}$ is directed upwards, $E_{\text {cas }}$ is the Casimir energy and the evaluation is performed to first order with respect to the quantity $\frac{\mathrm{ga}}{c^{2}}$. This force, directed upwards, can be interpreted as the lack of weight of the modes that have been removed by the cavity, in similarity with the Archimedes buoyancy of fluid. Notice that, as expected (the calculation relying upon the assumption that vacuum energy gravitates), the result is in agreement with the equivalence principle [4] and the force can also be interpreted as the effect of the gravitational field on the negative mass associated to the Casimir energy [5]. From an experimental point of view, the critical points are essentially two: 1) the expected force being quite small, the measurement must be performed modulating the effect; 2) a suitable device must be studied to perform the measurement. As far as the first point is concerned, as has been recently discussed [6], a possible way to modulate the vacuum energy contained in a cavity is by performing a superconducting transition of the plates: when the plates become superconducting the reflectivity changes and so it does the vacuum energy contained between the plates [7]. The variation of Casimir energy could be particularly relevant in case of type II layered superconductors, like cuprates. Even though a complete study is still lacking, and it is one of the problems to be faced inside the Archimedes project, order of magnitude expectations suggest that measuring the weight of the transition energy of such materials could be a test of the weighing of vacuum energy $[6,8]$. The amplitude $\mathrm{F}_{m}$ of the modulation of weight turns out to be quite small, of the order of $\mathrm{F}_{m} \approx 10^{-16} \mathrm{~N}$. This force is not out of reach of the presently most sensitive macroscopic devices for the measurement of small forces, such as the Gravitational Wave detectors or torsion pendulums [6]. In this paper we present the main choice exploited by the Archimedes project and discuss the steps to be done to assess the actual feasibility of an experiment for the final measure of such a force. 

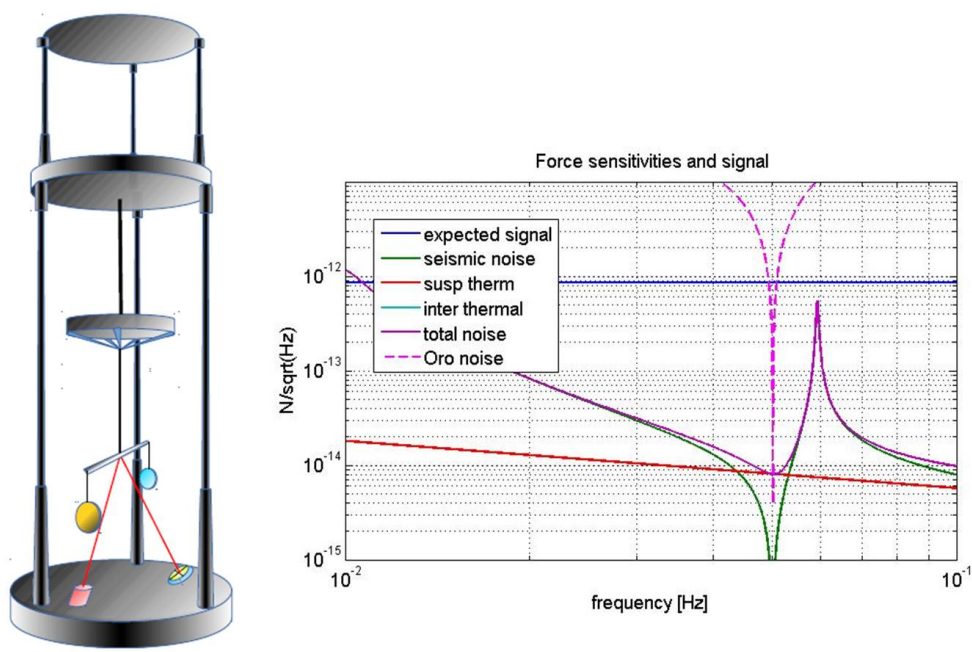

Figure 1: Experiment scheme, expected signal and noises. Left: scheme of the balance suspended to the inverted pendulum. Right: expected signal (blue curve) and main noises. The detection bandwidth is within the resonance of the balance, limited by the read-out noise, dashed-line, indicated as Oro Noise.

\section{Balance versus suspended masses interferometer}

In a recent paper [6] it has been shown that both the Gravitational Wave Detector and balances could be suitable for detecting the Archimedes force from a sensitivity point of view. In particular, the third generation GW detectors, i.e. the planned Einstein Telescope, could reach a sensitivity of about $\tilde{F}=3 * 10^{-15} \mathrm{~N} / \sqrt{(\mathrm{Hz})}$ in the low frequency region, corresponding to the detection of the Archimedes force in tens of minutes of integration time. In order to choose the detection system one experimental key point is the modulation of the effect, i.e. the periodical transition from normal to superconducting state. In particular, it must be compatible with the bandwidth of the detectors. The possible modulations of the transition are by temperature or external field. Both are favored in the low frequency region. This motivation leads us to the choice of the balances as the system to be experimentally used for the detection of the force. Indeed, in case of balances, the detection bandwidth can be in the region of 1-100 mHz, comparable with torsion pendulums, while in case of Gravitational Wave detectors it extends from $10 \mathrm{~Hz}$ to few KHz. The possibility to modulate at so small frequency is seen as a decisive argument in favour of using balances, even though in the long term the use of third generation GW detectors could be re-considered.

\section{Seismic isolation and expected sensitivity}

One of the main problems to be addressed in realizing a balance capable of measuring forces of the order of few $10^{-16} \mathrm{~N}$ is the lack of an attenuation system in the very low frequency regime of 1-100 mHz. The realization of such a system would allow the balance to reach a vertical force sensitivity compatible with the torsion pendulums horizontal one, which can be as well of the order of few $\left.10^{-15} \mathrm{~N} / \sqrt{(} \mathrm{Hz}\right)$ [9]. One possible strategy is to hang the balance to a seismic isolation cascade formed by an inverted pendulum and blade-spring attenuator similar to the ones used in the Virgo gravitational wave detector, and actively reduce the residual motion by sensing with 
accelerometers and feed-backing on the top of the cascade [6, 10]. An alternative reduction of residual noise is based on a mechanical resonator, placed on the top of the Inverted-Pendulum as sketched in fig. 1; the absorber is coupled to the Inverted-Pendulum so as to have the complex-zero of the transfer function tuned at the signal modulation frequency. In this way the seismic energy is absorbed by the resonator at that frequency and the suspension motion at the frequency is reduced by the attenuation transfer function of the Inverted-Pendulum and by the quality factor of absorber resonance. The major sources of noise in this scheme will be represented by the thermal noise and the seismic noise, as reported in fig. 1. The read-out system, as discussed in [6] and also reported in fig. 1, can be an optical lever. As shown in the figure, with these choices the modulation frequency, the seismic-resonant absorber frequency and the balance frequency must be carefully tuned to be the same, and forced to lie in the low-noise seismic region. Furthermore, the experiment must be performed in very low seismic noise sites, like the Sos-Enattos mine (Sardinia Island, Italy) [11]. These limitations should be compared with the use of an active seismic noise reduction scheme, that in principle does not require such a quiet environment and relaxes the constraints on the bandwidth, but at the price of a remarkably higher complexity [6]. The assessment of the passive reduction and comparison of these two methods will be one of the major tasks of the Archimedes experiment.

\section{References}

[1] S. Weinberg, Rev. Mod. Phys. 61 (1989) 1; M. Ishak, Found. Phys. 37 (2005) 1470.

[2] C. Rovelli, Quantum Gravity, Cambridge University Press, Cambridge (2004); E. Bianchi and C. Rovelli, Nature 466 (2010) 321; C. Kiefer, Quantum Gravity, Clarendon Press, Oxford, (2012); B.S. DeWitt and G. Esposito, Int. J. Geom Methods Mod. Phys. 5 (2008) 101.

[3] T. Padmanabhan, Int. J. Mod. Phys. D 15 (2006) 2029.

[4] S. A. Fulling et al, Phys. Rev. D 76 (2007) 025004; S. A. Fulling, K. A. Milton et al, J. Phys. A 40 (2007) 10935; K. A. Milton et al, J. Phys. A 41 (2008) 164052; K. V. Shajesh et al. J. Phys. A 41 (2008)164058 ; F. Sorge Class. Quant. Grav 26 (2009) 235002; K. A. Milton et al, Phys. Rev. D 89 (2014) 064027;

[5] E. Calloni et al, Phys. Lett. A 297 (2002) 328 ; G. Bimonte et al, Phys. Rev. D 74 (2006) 085011; G. Bimonte et al, Phys. Rev. D 76 (2007) 025008.

[6] E. Calloni et al, Phys. Rev. D 90 (2014) 022002.

[7] G. Bimonte et al, Phys. Rev. Lett. 94 (2005) 180402 ; G. Bimonte et al, Nucl. Phys. B 726 (2005) 441; G. Bimonte et al, J. Phys. A 41 (2008) 164023; A. Allocca et al, Jour. Super. and Novel Mag. 25 (2012) 2557.

[8] G. Barton, J. Phys. A 38 (2005) 2997 ; M. Bordag, J. Phys. A 39 (2006) 6173; A. Kempf, J. Phys. A 41 (2008) 164038.

[9] L. Carbone et. al, Class. Quant. Grav. 22 (2205) S509.

[10] J. Harms et al, Phys. Rev. D 88 (2013) 122003.

[11] L. Naticchioni et al, Class. Quant. Grav. 31 (2014) 105016. 